consumed. It may have been a small quantity, say 100 cubic centimetres, which each kitten drank. In this case the animal ingested 0.057 gramme or 0.88 of a grain of boric acid. The mean weight of the kittens in the series was 4084 grammes.

It must now be settled to what age in a child these threeweeks-old to four-weeks-old kittens are comparable. It re accept that a man grows till he is 20 years of age and that a cat grows till it is two years old, then the kittens experimented on may be compared to children of from nine to 10 months old. A child of this age has a weight of, it may be, eight kilogrammes and 300 grammes, and corresponding to the weight of the kittens such a child would require to receive 1.13 grammes or 1744 grains of boric acid. To obtain this it would be necessary for a child to use one litre and 983 cubic centimetres of the milk consumed by the kittens $(0.57$ gramme of boric acid in one litre). But such a child at the very utmost could only partake of one and a half litres per day, which would contain 086 gramme, or 13.25 grains, of boric acid. To make a general comparison with the experiments on the kittens, since the child has a weight of eight kilogrammes and 300 grammes and the kittens of 408.4 grammes, the kitten ought to have received no more than 0.042 gramme of boric acid, and this quantity must be contained in 100 cubic centimetres. This would correspond with milk containing only 0.42 gramme per litre or 29.45 grains per gallon, while as much as 40 grains per gallon was used in these kittens. This difference of more than 25 per cent. of the stated quantity of boric acid is very important, particularly when the treatment is continued over a long period. According to this calculation, made in a very favourable light from the experimenter's standpoint, it is evident that Dr. Annett is very far removed from having proved by his experiments that small quantities, if used for a long time, are poisonous for men and children.

\section{THREE CASES IN WHICH MOVEABLE KIDNEY PRODUCED ALL THE SYM- PTOMS OF GALL-STONES.}

BY T. J. MACLAGAN, M.D. EDIN., M.R.C.P. LOND., AND

FREDERICK TREVES, F.R.C.S.'ENG.

CASE 1.-A woman, aged 35 years, a widow with no children, tall and of spare habit, consulted me [Dr. Maclagan] in 1892. She had never had any serious illness, but for the last three years had suffered from occasional attacks of pain in the epigastrium, from indigestion and general loss of strength. She was thin and not well nourished her complexion was sallow; she was somewhat anæmic and had the expression of one who suffered a good deal. She said that she was never quite well, had always more or less discomfort in the abdomen, and every now and then had an attack of severe pain-so severe as to make her call out. These attacks lasted sometimes for an hour or two, sometimes for eight or 10 hours, and sometimes for a whole day. The pain was referred to the hepatic and epigastric regions. The attacks had of late become more frequent as well as more severe; she seldom went a week without one and was getting worn out by them. After the attacks she was generally slightly jaundiced for a few days. She had consulted various physicians, some of whom regarded the pain as neuralgic and others thought that she had gall-stones. She underwent a course of treatment at Schwalbach without deriving any benefit from it. She did the same at Carlsbad the following year. From this she said she derived some benefit; bat the excellent physician who looked after her tfere told her that she had a large gall-stone in her gall-bladder and that she would be no better till it was removed. When I saw her first she had considerabie discomfort but was not in pain. There was tenderness on pressure over the epigastrium and at the edge of the left lobe of the liver; the gall-bladder could not be felt. The right kidney was very moveable and could be distinctly felt under the edge of the liver. No other abnormality was found. A week later I saw her in one of ber attacks of pain. She was suffering much, the pain being referred to the epigastrium and to the right of it. The pulse was quick aud feeble and the temperature was $998^{\circ} \mathrm{F}$. She was tinder to the touch over the seat of pain and the gall-bladrler could be felt full and distended. The least pressure over it caused considerable pain. She had a dore of morphia and the pain passed off, leaving her slightly jaundiced for a few days. It was some days before the abnormal tenderness on pressure disappeared. A fortnight later she bad another attack in all respects similar, and within the following three months she had five or six more. During each attack the gall-bladder filled up and after each she was slightly jaundjced for two or three days. Trie kidney could always be detected under the border of the liver. The character of the attacks and the symptons produced all pointed to the presence of a gall-st ne in the gall-blarder small enough to get into the cystic duct but too large to pass through it. This was explained to the patient and an operation was recommended for the removal of the stone and fixing of the kidney. Mr. Knowsley Thr.rnton saw ber with we and concurred in this view. On May 5th, 1893, he performed the operation. He first, by an incision in front, got at the gall-bladder. Nothing was found amiss with it. The gall.bladder and gall-ducts were carelully examined but no gall-stone was found, but behind it was felt a lump which turned out to be the right kidney. The wound in front was closed by stitches. The kidney was then reached by incision in the lumbar region and secured to the side by three silk sutures. The wounds healed rapidly, the patient made a good recovery, and six weeks aft $r$ the operation went to the country to recruit. She remained well and has had no recurrence of her old symptoms.

CASE 2 -The patient was a woman, aged 34 years, the mother of three children. She had a history of having suffered from gall-stones for five or six years. On Nov. 17th, 1892, I saw her with Dr. J. Smith of Windsor. For two days she had been suffering from stvere pain in the epigastric and hepatic regions. pain which was kept down only by injections of morphia. Sbe was quite jaundiced, the temperature was $101^{\circ} \mathrm{F}$., and the tongue was dirty. The gall-bladder could be felt full and distended; it was very tender, but as she was a good deal under the influence of morphia there was not much difficulty in examining it. On the following day the pain abated and she was able to do without the morphia. $A$ gall-stone was found by the nurse in the stool. She remained jaundiced for a week or 10 days. She consulted me again in 1894. She said that she had never really been well since I saw her in 1892 She was constantly baving attacks of pain, seldom going more than a week without one. For the relief of these she took opium. She was sallow, thin, and had a look of ill-health. There was tenderness on pressure over the epigastrium and the region of the gall-bladder but no swelling or fulness. The right kidney was very moveable and could be felt under the edge of the liver. I dieted her, gave a mixture containing sulphate and bicarbonate of soda, and enjoined a quiet life. In 1895 she again consulted me for the same symptoms and received the same treatment. I did not see her again till the spring of 1897 when she came to London to be under my care. She told me that she was steadily getting worse, the attacks being more frequent and more severe, so much so that she seldom passed more than two or three days without having to take morphia for the relief of the pain. She was thin, badly nourished, and slightly jaundiced. There was tenderness on pressure over the epigastrium and over the region of the gall-bladder, but no distinct fulness. The right kidney could be felt as before under the liver and was very moveable. Though no gall-stone was found in the stools there seemed no doubt that every few days a stone was either passing or trying to pass. She was getting worn out by the pain and though in time the gall-bladder might empty itself this seemed too great a risk to run. I explained the circuinstances to the patient and to her husband and recommended an operation for the removal of the stones. Mr. Treves saw her with me and quite concurred both in my view of the case and in the advisability of operating. The patient was suffering so much and her life was such a burden to her that she readily assented. On May 26th, 1897, the operation was performed. Mr. Treves had better tell this part of the story:-"Immediately the abdomen was opened the gall-bladder presented. It was very much enlarged and was fully distended. It was readily emptied by pressure and was found to be free 
from gall-stones as were also the biliary ducts. There were no adhesions. On examining the neck of the gall-bladder the kidney was encountered. Its upper end was actually lying on the cystic dact and the gland had to be displaced before the ducts conld be examined. There was no evidence that the disturbance of the peritoneum attending the moveable kidney had led to such a mobility of the duodenum as could produce a kinking of the duct. The condition discovered definitely suggested that the kidney pressed directly upon the biliary passages. The gall-bladder was not opened and the abdominal wound was closed. An incision was then made in the loin and the kidney-which was very moveable-was secured by three silk sutures. The fact remains that the patient was cured of attacks of hepatic colic attended by jaundice by the fixing of her right kidney." The patient made an excellent recovery and went to the country six weeks after the operation. She has had no return of pain and now, two and a half years after the operation, ramains well. She has gained in flesh and looks the picture of health. I accidentally met her two years after the op eration and did not recognise her at first so much had her appearance changed for the better.

CASE 3.-A woman, aged 34 years, the mother of two children, of spare habit, had suffered for three or four years from indigestion, pain in the epigastrium, and symptoms of biliary catarrh. At times she had attacks of pain in the epigastrium and the region of the liver. During these attacks the gall-bladder could generally be felt more or less distended, and after each she was slightly jaundiced. The right kidney was moveable and could be felt under the liver. She improved at times under treatment, but never got well. During the winter 1897-98 matters got worse. Attacks of pain were more frequent as well as more severe. She lost flesh and was persistently slightly jaundiced. In the interval between the attacks there was more or less tenderness on pressure over the epigastrium; during them this tenderness was more marked, and, in addition, the gall-bladder could be felt full and distended, and was very tender. There was usually a slight rise of temperature. The diagnosis formed was that the gallbladder contained a gall-stone which was small enough to enter the cystic duct but too large to pass through it. I had the advantage of having the opinion of Sir William Broadbent and of Mr. Treves, who both took the same view. It was decided to go on treating her medically in the hope that the stone would yet pass. She seemed to improve a Iittle. In July, 1898, she went to Harrogate for a course of waters which seemed to do her much good. From there she went to America, where she spent the winter of 1898-99. There she got much worse, was constantly laid up with attacks of pain the same as before, only more severe. There the physician who attended her, Dr. Kinnicut of New York, treated her for gallstones. She returned to London in April, thin, slightly jaundiced, and much worn by suffering. She was scarcely ever free from pain and every few days had a severe attack calling for the use of morphia. The gall-bladder could now always be felt; during an attack it was very tender and could be felt, to use Mr. Treves's simile, "like a small banana" in the abdomen. The right kidney was still displaced and very moveable. After watching her for a few weeks I felt that I was not justified in temporising any longer. The gall-bladder was so permanently distended and so tender that the patient ran the risk of acute inflammation and suppuration there. Again Sir William Broadbent and Mr. Treves saw her with me. They quite agreed that there was distinct risk in allowing matters to go on and that the gall-bladder should be opened and the stone removed. The patient and friends readily consented and the operation was performed by Mr. Treves on June 30th, 1899. Again, as in the last case, no stone was found. What was found Mr. Treves shall describe. "An incision was made in the upper part of the right semilunar line. The gall-bladder presented and was not less than four times its normal size. It was opened by an incision made in the fundus. A considerable part of the body of the gallbladder was adherent to the great omentum. The bladder was emptied and I introduced my forefinger. On reaching the neck of the gall-bladder I encountered a tumour which pressed upon that part and which was rery readily felt through the anterior wall of the riscus. An examination with the disengaged hand showed that the 'tumour' was the npper end of the right kidney. There is no doubt whatever bat that the kidney was directly pressing upon the cystic duct. No gall stones were found. The opening in the gallbladder was closed by a double line of sutures and the abdominal wound was also closed. A second incision was made in the loin, and the right kidney, which was very moveable, was secured by three silk sutures. In this, as in the previous case, attacks of hepatic colic, attended by some janndice, were cured by suturing the right kidney." The patient went on well without any drawback for 12 days, when she was suddenly seized with great oppression of breathing, urgent cough, and bloody expectoration due to pulmonary embolism. She gradually recovered from this. The congh which persisted for some time was most unfortunate for the abdominal wounds, but notwithstanding it all went well and she left London for the country on August 8th, rather less than six weeks after the operation. Now, four months after it, she has a look of health, has a good colour bas gained considerably in flesh, and has had no return of her old pain. The only thing complained of is some pain and discomfort in the right side and extending down towards the groin. This no doubt is due to the stitches in the kidney and the drag on them.

The point of these three cases-the point which is specially desired to bring before the profession-is that all the symptoms of gall-stones-attacks of hepatic colic followed by janndice-may be produced by pressure on the bile ducts by a displaced right kidney. In each of these three cases the symptoms were exactly those produced by gall-stones and that the symptoms were due to that cause was the diagnosis formed by all the physicians and surgeons who saw them. In not one of them was a gall-stone found. In each the symptoms were due to the same cause, the pressure on the bile ducts of a displaced kidney, a pressure which was, from the nature of the disturbing cause, not continuous but occasional and intermitting-so mimicking exactly what occurs in gall-stones. In Cases 1 and 3 the pressure was on the cystic duct and the jaundice therefore was less marked and the pain probably less severe; in Case 2 the pressure was on the common duct and the jaundice more marked in consequence. It is to be noted, too, that this patient was said once to have passed a gall-stone which was found in the stool.

Literature.-Murchison thus enumerates the causes which may produce jaundice by pressure on the bile duct from without: (1) tumours projecting from the liver itself ; (2) enlarged glands in the fissure of the liver; (3) tumour of the stomach ; (4) tumour of the pancreas; (5) tumour of the kidney ; (6) post-peritoneal or omental tumour ; (7) an abdominal aneurysm; (8) accumulation of fæces in the bowels (9) a pregnant uterus ; and (10) ovarian and uterine tumours (Diseases of the Liver, p. 336). There is no mention of moveable kidney. But this must be added to Murchison's list. The recognition of this agency as an occasional factor in causing jaundice is recent, and by no means generally accepted. But that it has to be accepted and dealt with as a clinical fact is demonstrated by the cases now recorded.

There is little literature on the subject, first because it is only of recent years that displacement of the kidney has had its clinical importance recognised, and secondly, because it is the still more recent advance in abdominal surgery that has demonstrated how serious a cause of disturbance such displacement may be. Dr. Hale White has recorded a case of jaundice caused by the pressure of a moveable right kidney on the bile ducts in which the jaundiced condition was cured by fixing the displaced kidney. Dr. Cordier of Kansas has recorded a case of moveable kidney in which the gall-bladder was found to be distended and in which the patient was at times slightly jaundiced. "The enlargement of the gall-bladder could be made to disappear in a measure showing that there was not a permanent or complete obstruction of its duct. That no further mistakes be made in her case I made a small anterior opening over the gall-bladder and explored it and its duct for stones, but none were found. This incision was closed and the usual lumbar incision was made, and the moveable kidney was anchored with three silk sutures to the fascia and muscles. Since the operation all her gall bladder symptoms have disappeared, and her appetite, digestion, and assimilation have greatly improved, and she writes me that she feels better than she has done for years."

The peculiarity of the three cases now recorded is that all the symptoms of gall-stones-recurrent attacks of hepatic

1 Brit. Med. Jour., vol. i., 1ç2., p. 223.

2 American Journal of Obstetrics, 1896, vol. xxxiv., p. 532 
colic followed by jaundice-were produced by the displaced kidney. But for the operation undertaken for the relief of these symptoms the true cause of the disturbance would not have been recognised. Before the days of modern abdominal surgery these patients would have gone on indefinitely, leading lives of suffering and misery, with the constant risk of more serious trouble arising. The demonstration of the true canse of the disturbance is due to modern surgical advance. The point to be accentuated is that hepatic colic and janndice may be produced by the pressure on the bile-ducts of a displaced kidney.

\section{A CASE OF HÆMORRHAGIC ASCITES.}

BY THEODORE FISHER, M.D., M.R.C.P. LOND.,

PATHOLOGIST TO THE BRISTOL ROYAL INFIRMARY AND PHYSICIAN TO OUT-PATIRNTS TO THE BRISTOL HOSPITAI FOR SICK CHILUREN.

THE case of hæmorrhagic ascites published by Dr. G. S. Middleton in THE LaNCET of Nov. 11th, 1899, p. 1286, recalled to my mind the following case, a brief note of which may be interesting.

A woman, aged 38 years, a domestic servant, was admitted into the Bristol Royal Infirmary on Jan. 20th, 1898, under the care of Dr. A. B. Prowse, to whom I am indebted for kind permission to publish the case. Four weeks before admission after being out-of-doors one evening she had felt cold and could not keep warm even in bed. She bad been more or less " chilly" from that time. Two or three days after the onset of the cold sensation some swelling of the abdomen was noticed. Pain in this region quickly supervened and there was occasional vomiting. The bowels were constipated. The motions at first were of black colour but later became yellow. The urine from the onset was high. colonred and turbid. On admission to the Bristol Royal Infirmary the patient's abdomen was very distended and measured 37 inches in circumference. General tenderness was present and the greater part of the abdomen was resonant, but shifting dulness was present in the flanks. There was nothing noteworthy in the physical signs of the heart and lungs. The urine was high-coloured and there was abundant deposit of mucus and urates. Neither albumin nor sugar was present. The temperature was subnormal $\left(97^{\circ} \mathrm{F}\right.$.). The abdominal pain continued. On the $22 \mathrm{nd}$ the bowels were moved four times and the motions contained much blood and mucus. The patient died suddenly on the 24th during a severe attack of abdominal pain.

At the necropsy the patient was found to be a stout woman with very distended abdomen and some odema of the legs. The peritoneal cavity contained about 12 pints of deeply blood-stained fluid. The small intestine from the duodenum downwards was intensely congested and of a deep purplish colour. The congestion of the ascending colon was less marked and the transverse colon, descending colon, sigmoid flexure, and rectum were quite unaffected. There were numerous petechiæ dotted over the peritoneal coat of the congested intestines and in places thin flakes of exudation were present. The veins of the mesentery were greatly distended and full of blood-clot, none of which, however, appeared to be ante-mortem. The veins running over the surface of the stomach were similarly distended and full of blood-clot. The portal vein also was about donble its normal size and was full of blood-clot of blackish-purple colour, like that present in the other veins. The divisions of the portal vein throughout the liver were a'so greatly distended. The bepatic veins were abnormally full but costained no ante-mortem thrombi. There was nothing abnormal in the inferior vena cava. The mucous membrane if the stomach was free from congestion. The duodenum was injected, but the colour of its mucous membrane was pale compared with that of the jejunum and ileum. The congestion of these divisions of the small intestine was intense and a large quantity of blood was mixed with the fæces in their interior. The mucous membrane of the ascending colon was also congested but to a much slighter extent. The wide area of congestion in the small intestine was free from the sticky mucus seen corering patches of congestion present in acute enteritis. The mesenteric veins and arteries were opened but no antemortem thrombosis was present. Beyond the distension of the veins above mentioned the liver showed nothing rotewurthy. It weighed 48 ouncts. The splet was enlarged, weighed 16 ounces, but was not softer than normal. There was nothing abnormal in the suprarenal bodies, pancreas, or kidneys. Both pleural cavities contained ten ounces of clear fluid. The trachea, bronchi, and lungs showed nothing noteworthy, but there was a very large congested lymphatic gland, of the size of a small hen's egg, situated below the bifurcation of the trachea. The heart and pericardium were free from disease. Cultures were taken by Dr. J. O. Symes from the spleen and from the enlarged lymphatic gland, but only the bacillus coli communis was found.

The congestion of the - testines followed closely the diso tribution of the superior mesenteric vein and I expected to find thrombosis of that vein. I was, however, disappointed; the superior mesenteric vein contained merely soft, dark post-mortem clot and the portal vein showed distension fully equalling in degree that of the superior mesenteric vein. Yet to the naked eye the divisions of the portal vein in the liver were free from ante-mortem thrombus, as were also the bepatic veins, which I have seen thrombosed alone leading to great congestion of the liver. Neither bacilli nom other micro-organisms were seen in microscopic sections of the liver stained by Gram's method and with methylene blue and fuchsin, but the small divisions of the portal vein showed organising thrombi in their interior. Only one small piece of the liver was taken for histological parposes and the discovery of thrombi in one small area does. not prove that they were present throughout the substanceof the liver. But while such absolute proof is ranting it must be allowed that extensive thrombosis of the small divisions of the portal vein would in great measure explain the congestion of the small intestine and the ascites. Such thrombi, however, whether limited in extent or widespread, must have been merely one of the morbid evidences of more abstruse pathological factors. The failure to discover some definite micro-organism does not prove that it was not present. The history given by the patient of a "chilly" feeling at the onset suggests a rise of temperature, and a rise of temperature further suggests scme acute or subacute infection, and it is reasonable to suppose that such infection, if present, took place by the alimentary canal through some: article of food.

Clifton, Bristol.

\section{A CASE OF MULTILOCULAR CYSTIC: EPITHELIAL TUMOUR OF THE JAW ; EXCISION OF HALF THE LOWER JAW; RECOVERY.}

By G. P. NEWBOLT, M.B. DURH., F.R.C.S. ENG., HONORARY SURGEON, LIYRRPOOL BOYAL SOUTHERN HOSPITAI.

CASES of multilocular cystic epithelial disease of the jaw in which the tumour has attained a large size before removal are not very common. This case, therefore, may prove o? interest to the profession.

A woman, aged 66 years, a widow, consulted me in August, 1897, regarding a tumour of the lower jaw of a cystic nature which she said had been growing for 20 years. A few days later, the patient being placed nnder chlorotorm, I laid open the cysts and gouged the parts freely, hoping that an operation of this kind might give her relief for some time. About January, 1899, however, there was a decided increase in the size of the tumour, and on May 15th she came into the Liverpool Royal Sonthern Hospital for removal of the growth (Fig. 1). On the 16th I removed the left balf of the lower jaw with the tumour involving it. It was necessary to split the lip through, but I had very little difficulty in disarticulating the bone and the coronoid process came out with the growth intact, none of the cysts being opened. Hæmorrhage was profuse, bat there was no diffculty in checking it. The wound was closed, a drain being. left at the posterior angle. The patient made an excellent recovery and was able to be up and about the ward at the end of 10 days and the wound bealed soundly in three weeks (Fig. 2).

An examination of the parts removed showed that the tumour consisted of the lelt half of the lower jaw dis tended by cysts, which varied in size from a pin's heact 